\title{
Clinical efficacy and safety of single cycle rituximab as induction therapy for aggressive neuromyelitis optica spectrum disorder in a resource limited center: a preliminary study
}

\author{
Tien Lee Ong ${ }^{1,2}$, Shanthi Viswanathan', Sheila Ong ${ }^{1}$, Fu Liong Hiew ${ }^{1}$ \\ 'Department of Neurology, Hospital Kuala Lumpur, Kuala Lumpur 50586, Malaysia. \\ ${ }^{2}$ Neurology Unit, Medical Department, Hospital Sungai Buloh, Sungai Buloh 47000, Malaysia
}

Correspondence to: Dr. Tien Lee Ong, Neurology Unit, Medical Department, Hospital Sungai Buloh, Jalan Hospital, Sungai Buloh 47000, Malaysia. E-mail: ongtienlee@gmail.com

\begin{abstract}
How to cite this article: Ong TL, Viswanathan S, Ong S, Hiew FL. Clinical efficacy and safety of single cycle rituximab as induction therapy for aggressive neuromyelitis optica spectrum disorder in a resource limited center: a preliminary study. Neuroimmuno/ Neuroinflammation 2020;7:311-8. http://dx.doi.org/10.20517/2347-8659.2020.05
\end{abstract}

Received: 9 Jan 2020 First Decision: 4 Mar 2020 Revised: 14 Mar 2020 Accepted: 27 Apr 2020 Available online: 12 Jul 2020

Academic Editor: Athanassios P. Kyritsis Copy Editor: Jing-Wen Zhang Production Editor: Tian Zhang

\begin{abstract}
Aim: To analyse the efficacy of single dose rituximab (RTX) as induction therapy followed by conventional oral steroid-sparing agents (azathioprine, mycophenolate mofetil or methotrexate) in a cohort of patients with aggressive neuromyelitis optica spectrum disorder (NMOSD) without CD19, 20 and 27 biomarker testing.
\end{abstract}

Methods: A retrospective analysis of clinical outcomes in eight patients with aggressive NMOSD treated with one course of RTX induction therapy in the Neurology Department at Kuala Lumpur Hospital from 2005 to 2018 was performed. The effectiveness of the treatment was determined by the number of relapses, expanded disability status scale, annualized relapsed rates, and modified Rankin Scale both before and after treatment. B cell enumeration testing was done instead of CD19, 20 and 27 biomarker testing.

Results: There was a reduction in the mean annualized relapse rate from 4.7 to 0.5 attacks per year after treatment $(P=0.011)$. Mean expanded disability status scale and modified Rankin Scale values improved from 5.4 to 3.6 $(P=0.018)$ and 3.6 to $2.6(P=0.023)$, respectively. No patient developed any adverse effect.

Conclusion: Single-course RTX induction therapy regime may be an alternative therapeutic option in resource limited hospitals to suppress NMOSD disease activity in the short term as pulse induction therapy whilst awaiting the effectiveness of conventional steroid-sparing agents. Further prospectively designed studies are required to prove efficacy.

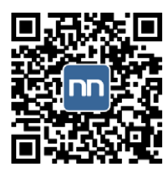


Keywords: Neuromyelitis optica spectrum disorder, single-course rituximab, aggressive neuromyelitis optica spectrum disorder

\section{INTRODUCTION}

Neuromyelitis optica spectrum disorder (NMOSD) is an autoimmune inflammatory demyelinating disorder of the central nervous system with preferential involvement of the optic nerve and spinal cord ${ }^{[1]}$. The disease has been reported worldwide with a prevalence ranging from $0.5-4.4 / 100,000^{[2]}$. In Malaysia, the crude prevalence rate of NMOSD is 1.9 per 100,000 population with a crude annual incidence of 0.3 per 100,000 population ${ }^{[3]}$. In this study, NMOSD patients notably experienced more relapses resulting in attack related disability, had higher expanded disability status scale (EDSS) scores and longer disease duration than MS patients ${ }^{[3]}$. If untreated, $80 \%$ of NMOSD patients experience a second attack within 2 years and the 5 -year mortality rate can be as high as $68 \%$ with $50 \%$ of the surviving patients developing permanent disability ${ }^{[4]}$. The mainstay of treatment for NMOSD consists of immunosuppressant therapy. In the acute management of relapses, corticosteroid and plasmapheresis are frequently used. However, long term use of corticosteroid often leads to multiple side effects ${ }^{[5]}$. Thus, conventional steroid-sparing immunosuppressive agents such as azathioprine (AZA), methotrexate and mycophenolate mofetil (MMF) are widely utilised as maintenance therapy to achieve remission and prevent relapses ${ }^{[4]}$.

Recent evidence points to B cell-mediated humoral immunity in the pathogenesis of NMOSD. Rituximab (RTX), a monoclonal antibody targeting the CD20 antigen on B cells, has been found to be effective in several modest quality studies in terms of reducing relapse rates and improving patients' outcomes ${ }^{[6-9]}$. RTX offers the prospect of an alternative steroid-sparing regime in the treatment of NMOSD, particularly when rapid disease control is required. Kim et al. ${ }^{[10]}$ demonstrated the benefits of induction RTX followed by repeated doses to control disease activity when CD27 and memory B cells in the blood increased to more than $0.05 \%$. Nevertheless, treatment with RTX is expensive and can cause profound B cell depletion, leading to the risk of severe immunosuppression and infection. Additionally, the monitoring of B cells and CD27 or CD19 are costly and multiple courses of RTX are a burden in regions with limited resources. At our institution, RTX is used as short course induction pulse therapy in patients with aggressive NMOSD followed by de-escalation to steroid-sparing agents such as AZA or MMF. Recent studies also suggest clustering of attacks occurring within 12 months of disease diagnoses, suggesting the hypothesis of "hitting hard" with early therapy to dampen the early aggressive inflammatory activity and later, down regulating to maintenance therapy may help in these fulminant situations ${ }^{[11]}$.

\section{METHODS}

In each patient, a diagnosis of NMOSD was made based on criteria described by the International Panel for Neuromyelitis optica (NMO) Diagnosis of $2015^{[1]}$. Relapses in this study were defined as objective worsening or new neurological symptoms and signs lasting for at least $24 \mathrm{~h}$. Aggressive NMOSD in our cohort of patients was defined as one or more disabling relapses in the preceding 12 months in patients with or without maintenance immunosuppressant therapy. Disabling relapses were defined as worsening EDSS at the time of relapse to 6.5 or more, Medical Research Council power of $3 / 5$ or worse in the lower limbs, or visual acuity worse than $6 / 36$ in one or both eyes at the time of relapse over the preceding one year. The patients in our observational study were on stable doses of oral prednisolone for at least three to six months prior to commencement of RTX.

This study was registered under the National Medical Research Register, Ministry of Health Malaysia (NMRR-19-291-46157 S1). 


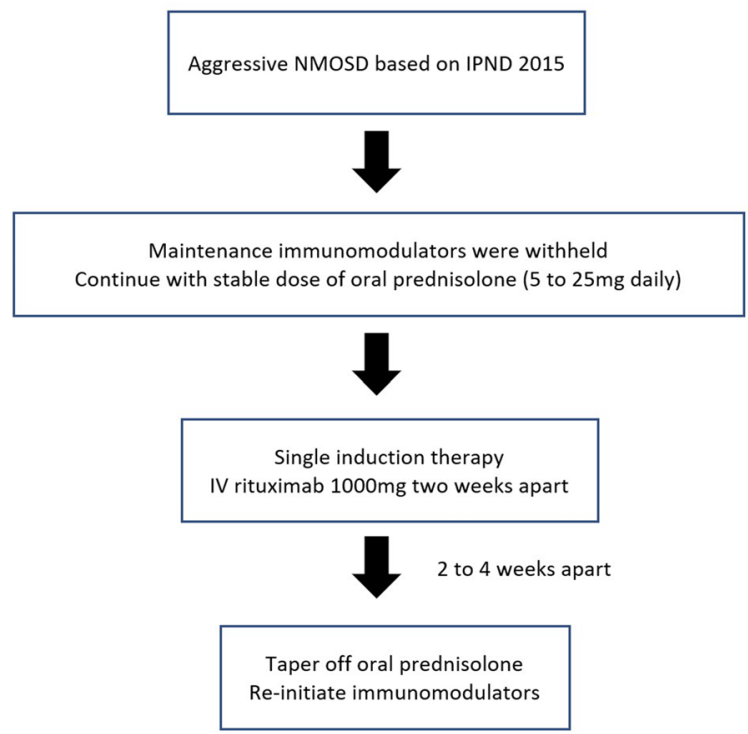

Figure 1. Protocol of single dose rituximab as induction therapy for aggressive NMOSD patients in Kuala Lumpur Hospital, Malaysia. NMOSD: neuromyelitis optica spectrum disorder; IPND: international panel for neuromyelitis optica diagnosis

This clinical outcome study is a retrospective analysis of patients with aggressive NMOSD treated with RTX in the Neurology Department at Kuala Lumpur Hospital from 2005 to 2018. The study included eight NMOSD patients who had received one course of RTX induction therapy. Clinical, laboratory and neuroimaging data from both in-patient admission and outpatient records of all patients were collected and analyzed. The primary outcomes included were the number of relapses, EDSS, annualized relapse rates (ARR), and modified Rankin Scale (mRS) before and after treatment. The EDSS was used to quantify the disability of our patients. It was assessed during six-monthly follow-up. ARR was defined as the number of clinical attacks per year. The secondary objective was to analyse the side effects of a single course rituximab treatment.

\section{Treatment protocol}

During the acute attack, high dose intravenous methylprednisolone at $1000 \mathrm{mg}$ daily was given for 5 days. Patients who continued to deteriorate or did not show significant improvement in clinical signs and symptoms after 2 weeks treatment were given plasma exchange (consisting of five cycles each). RTX was used thereafter for aggressive NMOSD patients who continued to have frequent relapses in a year while on oral immunosuppressant therapy.

All patients received RTX infusion as per our Department's protocol [Figure 1]. At the initiation of treatment, the medication was given as a slow intravenous (IV) infusion of two doses 2 weeks apart at $1000 \mathrm{mg}$ each. A similar regime was applied by Cree et al. ${ }^{[6]}$ and other RTX studies ${ }^{[5,12-14]}$. Patients were given premedication which consisted of IV promethazine $12.5 \mathrm{mg}$, IV hydrocortisone $100 \mathrm{mg}$ and oral acetaminophen $1000 \mathrm{mg}$ before the start of RTX infusion. All patients were then followed-up regularly at the Neurology outpatient clinic and assessed for further relapses, EDSS, mRS and side effects. Patients were monitored with full blood counts including lymphocyte counts and B cells (in selected patients), renal profile, liver function tests and C-reactive protein. Screening for chest infections were done with imaging and serum immunoglobulin levels were checked annually, if necessary.

While on RTX therapy, all patients had their pre-existing conventional steroid-sparing agents withdrawn and they were only re-initiated as per protocol 2 to 4 weeks after RTX induction therapy to prevent cumulative effects of infection or hematological and liver dysfunction amongst the patients. 


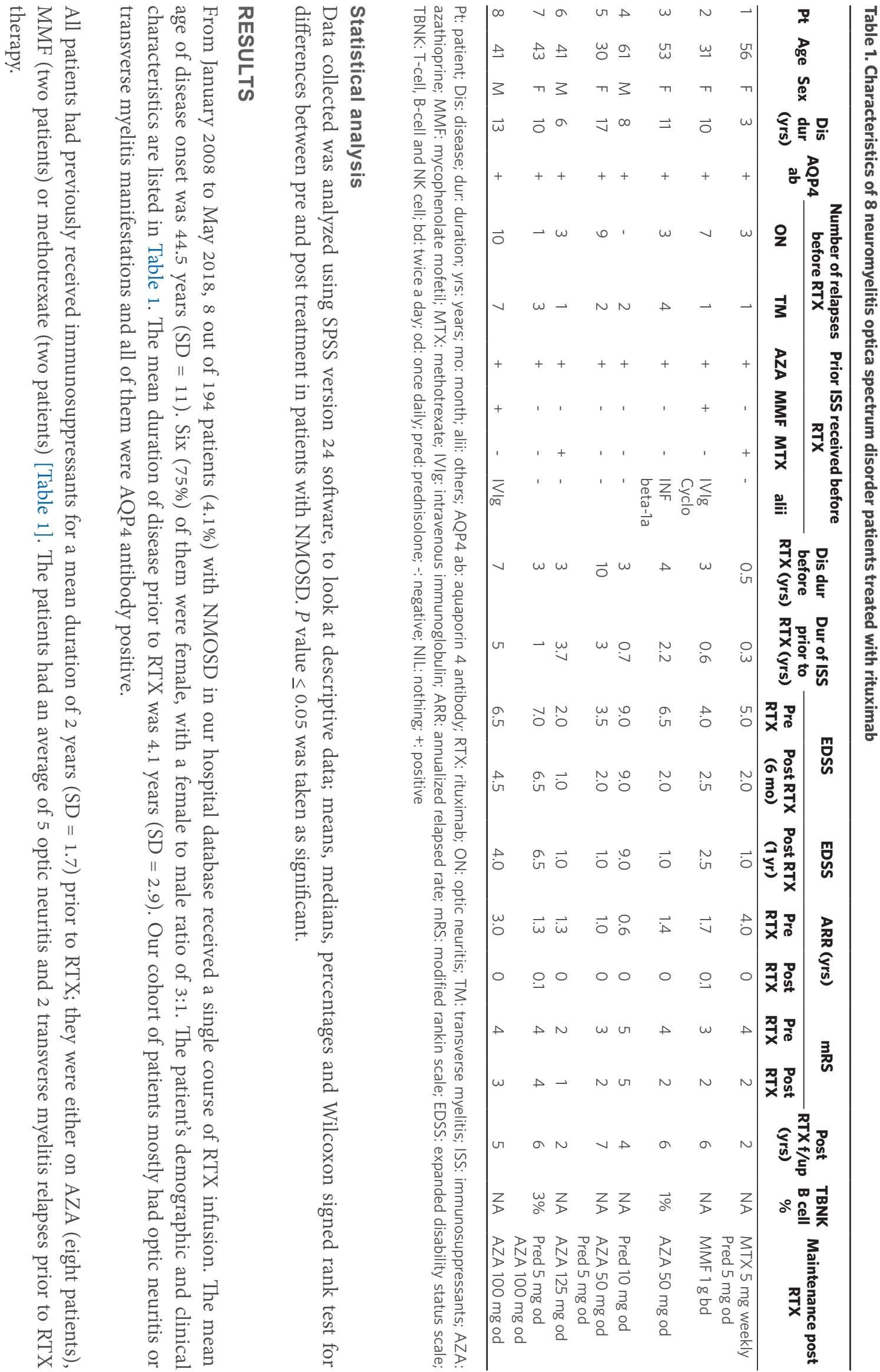




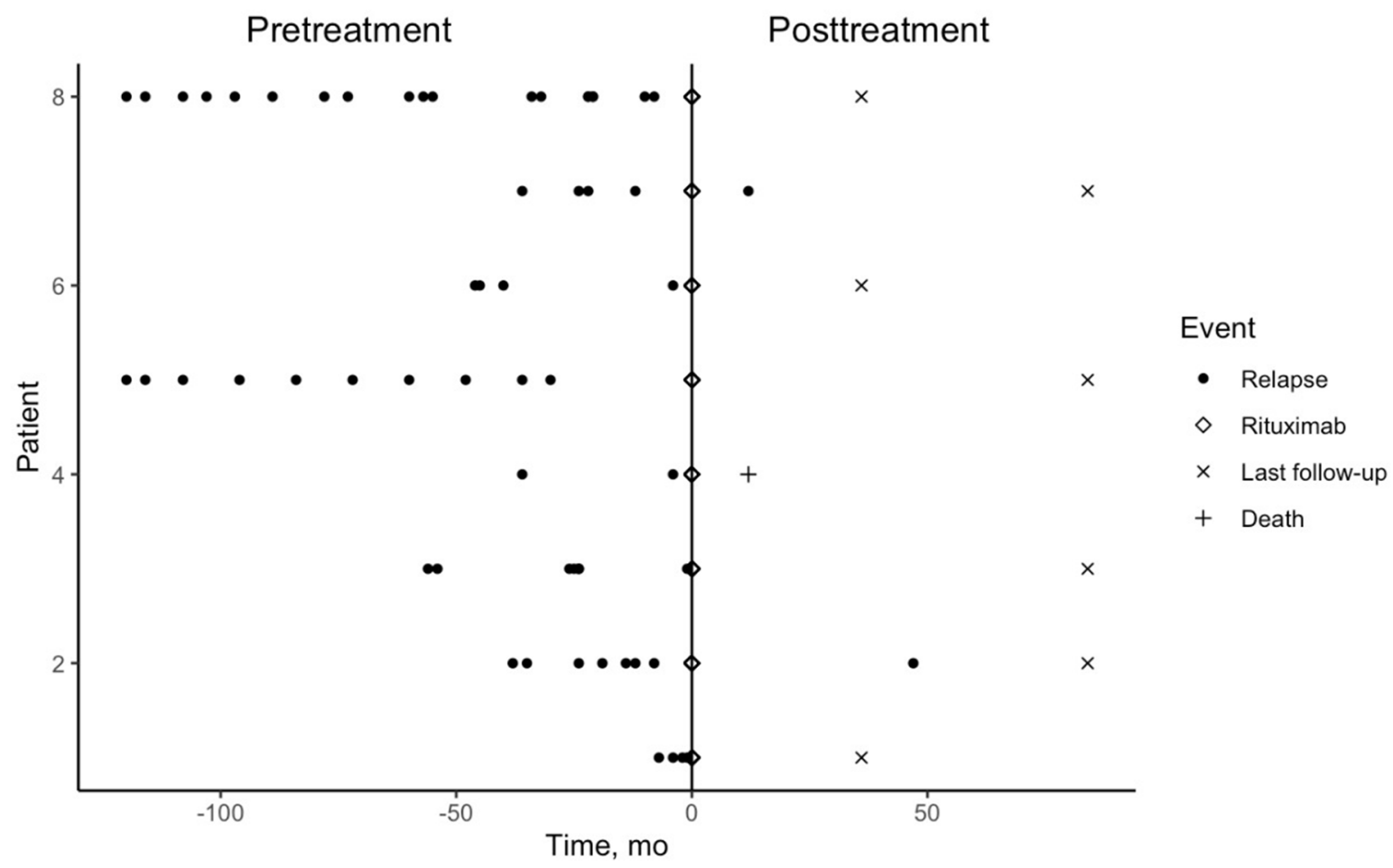

Figure 2. Relapses in patients with neuromyelitis optica spectrum disorder before and after treatment with rituximab. The mean follow-up duration after RTX therapy was 4.7 years. The use of rituximab was associated with significant reduction in the mean annualized relapse rate from 4.7 to 0.5 attacks per year after treatment $(P=0.011)$. Six patients $(75 \%)$ remained relapse-free during this period of follow up. Patient 2 suffered an attack of transverse myelitis, which occurred 4 years after the last dose of treatment due to poor compliance with maintenance oral immunosuppressants [Figure 1]. Patient 7 had a relapse five years after the last dose of rituximab therapy. This patient was on maintenance azathioprine from 2010 to 2016 when she developed transaminitis. Azathioprine was withheld, and she was on prednisolone $10 \mathrm{mg}$ daily when the disease relapsed

\section{Treatment efficacy}

The mean EDSS value prior to RTX treatment was 5.4 and after treatment, it improved to $3.6(P=0.018)$. Seven patients had reduction in EDSS scores whereas one patient (patient 4) did not show improvement following treatment. The same patient passed away due to myocardial infarction 1 year later [Figure 2]. The mean mRS prior to treatment was 3.6 and the mean mRS score post RTX improved to $2.6(P=0.023)$. On follow up, most of the patients following treatment were able to achieve independence in performing their activities of daily living.

\section{Safety profile}

Rituximab was well tolerated and none of our patients who received the therapy developed any shortterm adverse effects such as infusion related allergic reaction, fever, abdominal symptoms or hematological disorders. There was no reported case of progressive multifocal encephalopathy, malignancies, hypogammaglobulinemia or septicemia during the mean follow up period of 4.7 years ( 2 to 7 years) following RTX treatment.

\section{DISCUSSION}

In patients with NMOSD, recurrent clinical relapses can lead to rapid accumulation of disability and clustering of attacks. Aggressive immunosuppressive therapy remains the mainstay of NMOSD treatment to reduce the number of destructive demyelinating events. NMOSD is thought to be an aquaporinopathy that is predominantly humorally mediated, with complex interplay between B cells, T cells, complement and cytokines. Since the first open-label study evaluating RTX in NMO patients by Cree et al. ${ }^{[6]}$ in 2005, 
there have been several studies assessing the effectiveness of monoclonal antibody therapy, targeting CD20 epitope of the B cell lineage that is important in the pathogenesis of the disease ${ }^{[8,9,13,15-17]}$. In these studies, there was reduction in annualized relapse rates and improvement in disability as measured by the EDSS.

However, in our resource limited setting, RTX is expensive and obtaining the medication for our patients remains a huge challenge. Thus, we had to use a dosing schedule of lower frequency and a single course of RTX was applied followed by oral immunosuppression to treat these patients. Data collected retrospectively, showed a significant reduction in the frequency of relapses, ARR in NMOSD patients with aggressive disease especially in those failing conventional immunomodulators. Remission was also maintained in $75 \%$ of patients for 4 years. Similar to the study conducted by Bedi et al. ${ }^{[5]}$, we avoided abrupt withdrawal of oral prednisolone to prevent early relapses in those patients. Other immunomodulators were switched off while RTX was given. All our patients were on stable doses of oral prednisolone and they were either tapered off or reduced to the lowest possible maintenance dose of 5 to $20 \mathrm{mg}$ after subsequent doses of RTX were given, or when reasonable doses of conventional steroid-sparing agents was on board for an appropriate time limit without further disease relapse. Such an approach may have a positive effect on relapse control and contributed to the stability of disease activity after single dose RTX therapy.

To date, there has been no standard guideline or consensus on RTX treatment for patients with NMOSD. Existing protocols used to induce and maintain remission are characterized by heterogeneity in terms of infusion and monitoring schedules and methods. Previous studies practiced either prescheduled RTX induction regimen every 6 months, or retreatment based on B cell depletion monitoring ${ }^{[6-8,16-18]}$. CD19, $\mathrm{CD} 20$ and CD27 are among the commonly used treatment-related biomarkers ${ }^{[13,14,16,17]}$. However, our center lacks the facilities to monitor treatment with these tests though we are aware of the need to objectively test this for treatment response. We utilized lymphocyte enumeration tests by flow cytometry to estimate the $\mathrm{B}$ cell population as a guide to treatment. Nevertheless, the test is expensive and was only done in $50 \%$ of our patients who underwent RTX therapy. Thus, the data was too scarce to make a definite conclusion on retreatment decisions. Therefore, retreatment was guided mainly by the severity and frequency of ongoing attacks. In addition, our data demonstrated that eighty seven percent of our patients showed improvement in EDSS score and none of them required retreatment with RTX.

In resource-limited hospitals, a single induction course of RTX treatment may be a therapeutic and economic option to suppress disease activity. We recognized that oral prednisolone during RTX treatment may potentially confound the analysis, however the overlapping benefit of steroids with RTX may be crucial to avoid sudden withdrawal of immunosuppressants that can potentially cause an early relapse. The doses of steroids were kept stable during this period and other immunosuppressants were withdrawn to prevent possible adverse events.

Several studies have shown the benefits of low dose RTX in terms of improvement in ARR, disability scores and time to next relapse ${ }^{[17,19,20]}$. Kim et al. ${ }^{[17]}$ reported a less frequent RTX retreatment approach which could maintain remission in a subset of NMOSD patients. It is postulated that the initial high induction dose of RTX (IV $1000 \mathrm{mg} \times 2$ doses given 2 weeks apart) may be adequate to prevent early B cell repopulation, which is seen in some studies with lower initial doses of $\mathrm{RTX}^{[14,15]}$. To our knowledge, there has been no study to address the efficacy of a single course of RTX therapy followed by oral immunosuppressants maintenance in the management of aggressive NMOSD. In our small cohort of patients, regardless of the initial number of relapses and EDSS scores, majority responded very well to this treatment strategy and remained relapse-free.

Unlike the meta-analysis published by Damato et al. ${ }^{[21]}$, which recorded $26 \%$ of patients treated with RTX had adverse reactions, none of our patients exhibited any side effects. Specifically, Damato et al. ${ }^{[21]}$ reported 
that $10 \%$ of the patients experienced infusion-related adverse effects, but we managed to reduce this risk by premedicating our patients with IV promethazine, IV hydrocortisone and oral acetaminophen as stated in the treatment protocol. In addition, the absence of infection and leukopenia in our cohort of patients may be related to the withdrawal of immunomodulators prior to RTX and a delay of reinitiating the therapy for two to four weeks after treatment. We acknowledge that our small sample size might not reflect the actual safety profile of such an approach, however the absence of major side effects in our cohort of patients is promising for our therapeutic plan. We postulate that the lack of adverse events may be due to the absence of the cumulative effect of immunomodulators with RTX and the short frequency dosing as reported by others $^{[22]}$.

Nevertheless, there are several limitations to our study. Firstly, being a retrospective study, the analysis of data from medical records was subjected to recording bias. In addition, the patient group at baseline was heterogenous with regard to their pre-RTX status and have variable disease duration, number of relapses and EDSS severity. Oral immunosuppressant maintenance therapies and steroid doses following RTX treatment were also variable. Although our results were derived from a tertiary care institution, our sample size was small, which further highlights the challenges of access to RTX at tertiary establishments in a resource-limited setting.

In conclusion, pulse induction therapy with a single course of RTX followed by subsequent de-escalation to oral immunosuppressants may be a convenient and economical approach in managing NMOSD patients. In resource-limited hospitals with restricted access to RTX, such an approach can potentially be effective to reduce relapses and improve EDSS scores with minimal side effects. This treatment plan allowed adequate time for optimization of other oral medications to achieve their therapeutic benefits. Moreover, the ability to achieve and maintain remission suggests that RTX has long-term effects extending beyond treatment discontinuation. Nevertheless, we concede that a further, larger prospective cohort study is required to demonstrate the efficacy of such a treatment approach.

\section{DECLARATIONS}

\section{Acknowledgments}

The authors would like to thank the Director General of Health Malaysia for the permission to publish this paper.

\section{Authors' contributions}

Performed data acquisition, analysis and interpretation, prepared the manuscript, Tables and Figures: Ong TL

Made substantial contributions to the conception and design of the study and performed data analysis and interpretation: Viswanathan S

Performed data acquisition, analysis and interpretation. Contributed in manuscript preparation: Ong S, Hiew FL

\section{Availability of data and materials}

All data used for this study were collected as part of ongoing work under the auspices of the Demyelinating Disease Database, established at the Neurology Department of Kuala Lumpur Hospital.

\section{Financial support and sponsorship}

None.

\section{Conflicts of interest}

All authors declared that there are no conflicts of interest. 


\section{Ethical approval and consent to participate}

Informed consent, either verbal or written, was obtained from patients for the use of retrospective data with longitudinal follow-up for this study.

\section{Consent for publication}

Not applicable.

\section{Copyright}

(c) The Author(s) 2020.

\section{REFERENCES}

1. Wingerchuk DM, Hogancamp WF, O’Brien PC, Weinshenker BG. The clinical course of neuromyelitis optica (Devic's syndrome). Neurology 1999;53:1107-14.

2. Marrie RA, Gryba C. The incidence and prevalence of neuromyelitis optica: a systematic review. Int J MS Care 2013;15:113-8.

3. Viswanathan S, Wah LM. A nationwide epidemiological study on the prevalence of multiple sclerosis and neuromyelitis optica spectrum disorder with important multi-ethnic differences in Malaysia. Mult Scler 2019;25:1452-61.

4. Ganesh A. Practice current: how do you treat neuromyelitis optica? Neurol Clin Pract 2017;7:170-8.

5. Bedi GS, Brown AD, Delgado SR, Usmani N, Lam BL, et al. Impact of rituximab on relapse rate and disability in neuromyelitis optica. Mult Scler 2011;17:1225-30.

6. Cree BA, Lamb S, Morgan K, Chen A, Waubant E, et al. An open label study of the effects of rituximab in neuromyelitis optica. Neurology 2005;64:1270-2.

7. Cabre P, Mejdoubi M, Jeannin S, Merle H, Plumelle Y, et al. Treatment of neuromyelitis optica with rituximab: a 2-year prospective in multicentre study. J Neurol 2018;265:917-25.

8. Jacob A, Weinshenker BG, Violich I, McLinskey N, Krupp L, et al. Treatment of neuromyelitis optica with rituximab: Retropective analysis of 25 patients. Arch Neurol 2008;65:1443-8.

9. Greenberg BM, Graves D, Remington G, Hardeman P, Mann M, et al. Rituximab dosing and monitoring strategies in neuromyelitis optica patients: creating strategies for therapeutic success. Mult Scler 2012;18:1022-6.

10. Kim SH, Kim W, Li XF, Jung IJ, Kim HJ. Repeated treatment with rituximab based on the assessment of peripheral circulating memory B cells in patients with relapsing neuromyelitis optica over 2 years. Arch Neurol 2011;68:1412-20.

11. Akaishi T, Nakashima I, Takahashi T, Abe M, Ishii T, et al. Neuromyelitis optica spectrum disorders with unevenly clustered attack occurrence. Neurol Neuroimmunol Neuroinflamm 2019;7:e640.

12. Pellkofer HL, Krumbholz M, Berthele A, Hemmer B, Gerdes LA, et al. Long term follow up of patients with neuromyelitis optica after repeated therapy with rituximab. Neurology 2011;76:1310-5.

13. Fernandez-Megia MJ, Casanova-Estruch B, Perez-Miralles F, Ruiz-Ramos J, Alcala-Vincente C, et al. Clinical evaluation of rituximab treatment for neuromyelitis optica. Neurologia 2015;30:461-4.

14. Lindsey JW, Meulmester KM, Brod SA, Nelson F, Wolinsky JS. Variable results after rituximab in neuromyelitis optica. J Neurol Sci 2012;317:103-5.

15. Lp VH, Lau AY, Au LW, Fan FS, Chan AY, et al. Rituximab reduces attacks in Chinese patients with neuromyelitis optica spectrum disorders. J Neurol Sci 2013;324:38-9.

16. Jade JD, Bansi S, Singhal B. Rituximab in neuromyelitis optica spectrum disorders: our experience. Ann Indian Acad Neurol 2017;20:229-32.

17. Kim SH, Kim Y, Kim G, Park NY, Jang HM, et al. Less Frequent Rituximab Retreatment Maintains Remission of Neuromyelitis Optica Spectrum Disorder, Following Long-Term Rituximab Treatment. J Neurol Neurosurg Psychiatry 2019;90(4):486-7.

18. Lin J, Li X, Xue B, Tong Q, Chen Z, et al. Low-dosage of rituximab in Chinese patients with neuromyelitis optica spectrum disorder. J Neuroimmunol 2018;317:1-4.

19. Yang CS, Yang L, Li T, Zhang DQ, Jin WN, et al. Responsiveness to reduced dosage of rituximab in Chinese patients with neuromyelitis optica. Neurology 2013;81:710-3.

20. Danés I, Agustí A, Vallano A, Martínez J, Alerany C, et al. Available Evidence and Outcome of Off-Label Use of Rituximab in Clinical Practice. Eur J Clin Pharmacol 2013;69:1689-99.

21. Damato V, Evoli A, Iorio R. Efficacy and safety of rituximab therapy in neuromyelitis optica spectrum disorders: a systematic review and meta-analysis. JAMA Neurol 2016;73:1342-8.

22. Novi G, Bovis F, Capobianco M, Frau J, Mataluni G, et al. Efficacy of different rituximab therapeutic strategies in patients with neuromyelitis optica spectrum disorders. Mult Scler Relat Disord 2019;36:101430. 